Didáctica. Lengua y literatura

ISSN: 1130-0531

http://dx.doi.org/10.5209/DIDA.57131

\title{
Cincuenta palabras generatrices para el aprendizaje del código escrito de la lengua española. Adaptación del método de Paulo Freire a la edad infantil
}

\author{
María Luisa García Rodríguez¹ Noemí García Díaz
}

Recibido: 2 de julio de 2015 / Aceptado: 7 de julio de 2017

Resumen. Con el fin de facilitar el proceso de enseñanza-aprendizaje de la lengua española -en su versión escrita- para la enseñanza inicial en la infancia, se propone una adaptación del método de palabras generatrices creado por el gran pedagogo y educador brasileño del siglo XX, Paulo Freire. Además de inscribirse en su línea de pensamiento, dicha propuesta considera las vías de procesamiento de la lengua escrita y se enmarca en los planteamientos de la Corriente Psicolingüística, sin dejar de lado las aportaciones del Constructivismo. La selección de palabras se ha llevado a cabo mediante una investigación cualitativa a partir de la observación del vocabulario de alumnado de 4 a 7 años escolarizado en centros de educación formal de Salamanca capital.

Palabras clave: lengua española; método de aprendizaje; código lingüístico escrito; edad infantil; palabras generatrices.

\section{[en] Fifty words to learn the written code of the Spanish language. Adaptation of Paulo Freire'smethod to childhood}

Abstract. An adaptation of the method based on generating words by Paulo Freire, the well-known, 20th Century, Brazilian pedagogue and educator, is proposed in order to ease the teaching-learning of written Spanish in the beginning stages of early childhood. Not only does this proposal adhere to his thought and philosophy, it also takes into consideration the different ways of processing written language framed within the Psycholinguistic School proposals, without neglecting the Constructivism's contributions. The choice of words has been elaborated through qualitative research from the observation of schoolchildren' vocabulary from 4 to 7 year-old in the capital city of Salamanca.

Keywords: Spanish language; learning method; written linguistic code; childhood; generative words.

[fr] Cinquante mots générateurs pour l'apprentissage du code écrit de la langue espagnole. Adaptation de la méthode de Paulo Freire à l'âge enfantin

Résumé. Dans le but de faciliter le procès d'enseignement-apprentissage de la langue espagnole -à l' écrit - pour l'enseignement initial lors de l'enfance, nous proposons une adaptation de la méthode

\footnotetext{
Dpto. Didáctica, Organización y Métodos de investigación

Universidad de Salamanca

malugaro@usal.es

2 Universidad de Salamanca

noemigardi3@usal.es
} 
de mots générateurs créée par le grand pédagogue et éducateur brésilien du XX siècle, Paulo Freire. En plus de s'inscrire dans sa ligne de pensée, ladite proposition considère las voies de procédure de la langue écrite et s'encadre parmi les approches du Courant Psycholinguistique, sans laisser de côté les apports du Constructivisme. La sélection des mots a été faite à travers une recherche qualitative à partir de l'observation du vocabulaire des élèves âgés entre 4 et 7 ans inscrits dans des établissements d'éducation formelle de la capitale de Salamanca (Espagne).

Mots-clés: langue espagnole; méthode d'apprentissage; code linguistique écrit; âge enfantin; mots générateurs.

Sumario: 1. Introducción. 2. Paulo Freire: Aproximación al pensamiento del autor. 3.El método de las palabras generatrices de Freire entre los métodos de aprendizaje inicial de la lengua escrita española. 4.Adaptación del método de las palabras generatrices para la enseñanza de la lengua española.5.Nueva propuesta didáctica del método de las palabras generatrices.6.Consideraciones finales.7.Bibliografía. Anexo.

Cómo citar: García Rodríguez, M.L.; García Díaz, N. (2017). Cincuenta palabras generatrices para el aprendizaje del código escrito de la lengua española. Adaptación del método de Paulo Freire a la edad infantil. Didáctica. Lengua y literatura, 29, 2017, 81-102.

«Estaré preparando tu llegada como el jardinero prepara el jardín para la rosa que se abrirá en la primavera» Freire (De "Canción obvia", 1971)

\section{Introducción}

La cita de Freire constituye una sugerente evocación de la ilusión ante expectativa de la figura educadora al emprender la emocionante experiencia de acompañar el proceso de aprendizaje de la lengua escrita. Nos acercamos respetuosamente a la principal fuente de inspiración para redactar las páginas siguientes: la obra que Freire nos dejó escrita el siglo pasado y que sirve para contextualizar nuestro trabajo.

El desenvolvimiento en nuestra sociedad alfabetizada requiere el adecuado dominio de la lengua escrita, como recurso cotidiano y requisito imprescindible para garantizar la vida democrática, y como base para poder acceder a otros conocimientos, por lo que constituye uno de los pilares esenciales del currículo escolar.

La finalidad de estas páginas es presentar una nueva propuesta para la enseñanza de la lengua escrita española utilizando el método de las palabras generatrices de Freire y respetando el valor dialógico del método original para conseguir aprendizajes significativos y funcionales. Se parte de los intereses de quienes están en el periodo más oportuno para aprender la lengua escrita, que, en nuestro entorno cultural, es la edad de entre los 5 y los 7 años.

Para enfrentarnos a un texto escrito, numerosos estudios de la Corriente Psicolingüística han demostrado que utilizamos dos vías o rutas de procesamiento (Domínguez y Cuetos Vega, 1992; Clemente Linuesa, 2008): la vía directa o léxica, mediante la cual se reconoce la palabra a nivel global, por lo que se emplea para leer palabras familiares o conocidas que están en nuestro léxico o vocabulario interno; y la vía indirecta o fonológica, que requiere de un mecanismo de descodificación, con- 
sistente en ir asociando cada grafía con su fonema/sonido para acceder al significado de la palabra y que se pone en práctica al encontrarse con vocablos desconocidos (Domínguez y Cuetos Vega, 1992).

Asociar cada grafía con su sonido y realizar sus combinaciones supone al comienzo un gran esfuerzo y demanda mucha ayuda. El aprendizaje de este proceso requiere intervención didáctica sistemática por parte de profesionales especialistas, puesto que las habilidades metafonológicas (uso de la vía indirecta) no se desarrollan espontáneamente (Alegría, 2006).

Defender que el aprendizaje de la lengua escrita consiste en una "construcción" por parte de la mente infantil, que absorbe a su modo el código escrito utilizado en su entorno, con el estímulo y apoyo de la figura educadora, entronca con las aportaciones de la Corriente Constructivista, representada, entre muchas otras, por las investigaciones de Ferreiro y Teberosky (1979).

Para afrontar la enseñanza de la lengua escrita, se puede optar por diferentes métodos, que se diferencian, fundamentalmente, por la estructura gramatical de la que parten. Se distinguen dos tipos: los métodos sintéticos basados en el aprendizaje explícito de la correspondencia grafía-fonema, es decir, de cada letra con su sonido, para ir avanzando hasta estructuras más complejas (sílabas, palabras, frases, etc.), y los métodos analíticos cuya intencionalidad es potenciar el significado de los mensajes, por lo que se parte de la palabra o unidades superiores, para descender al conocimiento de las unidades más simples: sílabas y fonemas.

$\mathrm{Al}$ no existir un método universal y eficaz que responda a las características personales de la infancia, articuladas con las peculiaridades de la lengua, actualmente, los métodos sintéticos y analíticos, con sus ventajas y limitaciones (García Rodríguez y Sánchez Gómez, 2012), se combinan dando lugar a métodos mixtos, que tratan de superar el aprendizaje mecánico y memorístico de los fonemas propio de los métodos sintéticos, para dotar el proceso de aprendizaje de funcionalidad y significado desde el principio, ventaja que abanderan los métodos analíticos.

\section{Paulo Freire: aproximación al pensamiento del autor}

Aunque en el mundo de la educación es innecesario presentar al educador más novedoso del siglo XX, recordemos que Paulo Freire (1921-1997), brasileño, fue el defensor de las clases oprimidas. Creó y transmitió una pedagogía de la esperanza. Sus ideas enlazaron con innovaciones escolares europeas e inspiraron procesos democráticos en toda la Tierra. Con su principio del diálogo mostró un nuevo estilo de relación entre profesorado y educandos. Refiriéndose a la figura del educador humanista expresaba que "exige que sea, en sus relaciones con los educandos, un compañero de éstos" (Freire, 1984, 82).

Freire, consciente de la atmósfera opresora de las clases dominantes, que alienaba y sometía a las clases obreras, se rebeló contra un sistema educacional en el que primaba la pasividad del educando y el conocimiento memorizado. Criticó la educación calificándola de bancaria (entendida como almacenamiento de saberes), domesticadora o utilitaria, que responde al modelo de figura educadora que sabe, piensa y elige programas dirigiéndose a personas que no saben, escuchan dócilmente y reciben aquello que quien les educa tiene a bien darles. 
Su propuesta consiste en que educador y educandos, como sujetos activos del proceso de búsqueda de conocimiento y creación, confíen unos en otros generando un clima de familiaridad, en el que compartan su visión y sus reflexiones sobre el mundo en el que están insertos, con el fin de mejorar su situación actuando sobre ella (Freire, 2002).

De este modo, se da funcionalidad y sentido a la alfabetización, pues se parte del contexto concreto de los educandos y se generan situaciones de debate que les permiten llegar a una mayor comprensión de esa realidad y de la necesidad de alfabetizar separa cambiar positivamente su situación (Freire, 1984).

En definitiva, la pedagogía de Freire no se reduce a la dimensión metodológica (a la que nos ceñimos en estas páginas), sino que tiene una intención más amplia, que abarca las dimensiones filosófica y política. Alfabetizar para Freire es concienciar ("despertar la conciencia") y la educación se basa en el diálogo, es decir en la palabra como herramienta para transformar la realidad.

\section{El método de palabras generatrices de Freire entre los métodos de aprendizaje inicial de la lengua escrita española}

En consonancia con sus inquietudes políticas y sociales, Paulo Freire ideó un método psicosocial para la alfabetización de personas adultas: el método de palabras denominadas"generatrices", destacando que «como todo buen método pedagógico, no pretende ser un método de enseñanza, sino de aprendizaje», centrado en el alumnado (Freire, 2002, 21).

El método de las palabras generatrices creado por Freire es un método mixto analítico-sintético, puesto que parte de la palabra como unidad mínima con sentido completo para analizarla descomponiéndola en sílabas y letras (fase analítica), que posteriormente se van uniendo para construir nuevas unidades con significado (fase sintética).

Para conectar con los intereses de los educandos y dotar de significado el proceso educativo, este se inicia con la búsqueda del vocabulario conocido por el grupo de aprendices, pues considera fundamental tener en cuenta las peculiaridades del contexto y de las personas, tomando como palabras generatrices aquellos vocablos que son cercanos para quienes han de aprender. De este modo, potencia el protagonismo y la participación del alumnado, mostrándose contrario a planificaciones totalmente establecidas por el profesorado.

En su libro La educación como práctica de la libertad, define las palabras generatrices de la siguiente forma: «son aquellas que, descompuestas en sus elementos silábicos, propician, por la combinación de esos elementos, la creación de nuevas palabras» (Freire, 2002,109) y desarrolla las fases de elaboración y acción del método; que se exponen a continuación como marco teórico para que sea posible comprender mejor la propuesta didáctica que se presenta posteriormente.

\section{A: FASE DE ELABORACIÓN:}

1.Obtención del vocabulario conocido por el grupo de personas que han de aprender el código lingüístico: investigación, en encuentros informales, de las palabras más significativas para los propios educandos, vinculadas a sus vivencias personales. 
2.Selección del vocabulario identificado: Freire sometió la selección de las palabras generatrices a tres criterios:1) la riqueza fonética, es decir, palabras que contemplen todos los fonemas de la lengua española; 2) la dificultad fonética, para secuenciar las palabras en orden de dificultad creciente considerando la complejidad fonética de la lengua, y 3 ) el sentido pragmático de la palabra, esto es, interesa que sean significativas y con gran potencial de concienciación.

3.Creación de situaciones existenciales típicas del grupo de trabajo: A través de las palabras generatrices (graduadas según el criterio de su dificultad fonética) induce en los educandos la reflexión sobre situaciones-problema de la realidad, codificadas en imágenes. Se abre un debate en el que se analizan problemas nacionales y regionales, de forma que el grupo va concienciándose a la vez que se alfabetiza.

4.Elaboración de fichas de ayuda: que sirven de apoyo a "los coordinadores" (denominación que Freire utiliza para referirse a las figuras educadoras).

5.Preparación de fichas con las familias fonéticas de las palabras generatrices: Los coordinadores elaboran carteles de cada palabra generatriz y sus familias silábicas.

\section{B: FASE DE EJECUCIÓN PRÁCTICA:}

Se aborda proyectando una situación codificada mediante una representación gráfica y se debate sobre las repercusiones de dicha situación. Se presenta la palabra generatriz junto al objeto representado y se establece su correspondencia.

Posteriormente, se muestra la palabra generatriz sola (sin el objeto nombrado) y más tarde separada en sílabas. Una vez familiarizados con las sílabas, se presentan las familias fonéticas, primero de forma aislada y luego todas juntas (identificando las cinco vocales repetidas en todas ellas), de forma que los educandos descubren el mecanismo para formar nuevos vocablos combinando fonemas consonánticos con otros vocálicos. De este modo, desde el primer día, los aprendices comienzan a formar palabras combinando las familias fonéticas de la palabra generatriz.

Finalmente, escriben todos los vocablos creados, aunque no tengan significado, ya que el objetivo de esta primera tarea es que comprendan la lógica combinatoria para crear nuevas palabras. A continuación, con la ayuda docente, se clasifican en "palabras de pensamiento" o con significado y "palabras muertas" o sin sentido (que no existen en español), y se dialoga en grupo sobre las palabras de pensamiento recién creadas.

En la Figura 1, se muestra una visión general de las fases prácticas del método, expuestas por Freire.
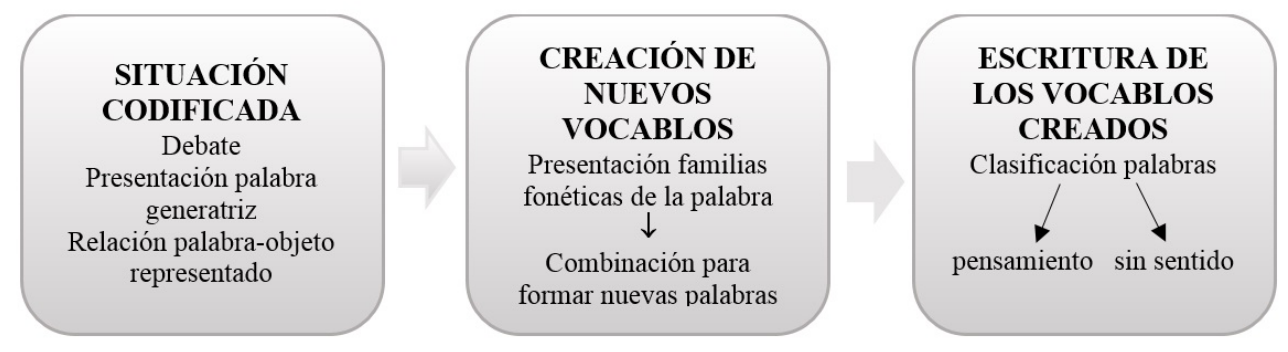

Figura 1: Fases de ejecución práctica del método de las palabras generatrices de Freire. Elaborado por García Rodríguez y García Díaz (2017) 


\section{Adaptación del método de palabras generatrices para la enseñanza de la lengua española}

Al estar basada en la práctica, la pedagogía de Freire está sometida constantemente al cambio, a la evolución dinámica y a la reformulación. Siguiendo sus pasos, Santos y Mateos (1985) realizaron una adaptación para enseñar la lengua escrita española en las escuelas de la ciudad de Salamanca, constituyendo esta adaptación nuestro referente más directo, al no haber encontrado otra versión posterior.

En dicha obra se definen las palabras generatrices como «vocablos muy significativos por su pertenencia al universo psicosocial del niño» (Santos y Mateos, 1985, 17), de lo que se infiere que también comenzaron investigando el vocabulario de la población infantil salmantina. Adoptaron el orden de adquisición de consonantes propuesto por Rondal en 1982 y declararon que basaron la selección de palabras en dos criterios: la riqueza silábica y el orden de dificultad creciente de las consonantes que integran la palabra.

La relación de palabras generatrices propuesta por Santos y Mateos (1985) consta de sesenta y dos palabras, estructuradas en seis grupos secuenciados según el criterio de la complejidad de la estructura silábica (de menor a mayor dificultad): sílabas directas, inversas, mixtas, trabadas, palabras con "y" como semivocal y diptongos.

Manteniendo como premisa básica la pedagogía dinámica de Freire, tras la experiencia en el aula infantil con este método de una de las autoras de estas páginas, se estima que, más de tres décadas después de la adaptación realizada por Santos y Mateos, es pertinente elaborar una nueva propuesta de palabras generatrices, ya que ha evolucionado el contexto y, paralelamente, el vocabulario infantil.

\subsection{Exploración del vocabulario de la población infantil salmantina actual}

Se plantea así la necesidad de investigar el vocabulario de la población infantil actual de la ciudad de Salamanca con el objetivo de seleccionar un conjunto de palabras significativas, pertenecientes a su entorno próximo y afines a sus intereses, que favorezcan el diálogo sobre la realidad que representan y fomenten el interés por aprender a leer y escribir. Se atenderá a la vez a criterios psicolingüísticos.

\subsubsection{Tipo de estudio}

Metodológicamente se opta una investigación cualitativa por su flexibilidad para profundizaren la didáctica de la lengua escrita, permitiendo aportar una nueva propuesta.

Como principal estrategia de recogida de datos se utiliza la observación directa y sistemática de la población infantil salmantina. El procedimiento de observación empleado es el muestreo ad líbitum, que consiste en registros no sistematizados o notas de campo de las palabras altamente significativas para los niños y niñas de 4 a 7 años, que hacen referencia a sus intereses y experiencias vitales.

Para cumplir también con el criterio de suficiencia que debe darse en una investigación cualitativa (Rodríguez Gómez, Gil Torres y García Jiménez, 1996, 75) y para incluir un conjunto de palabras que contengan todas las grafías de la lengua castellana, se han tomado algunas palabras de la colección de treinta y dos cuentos 
El zoo de las letras (Doumerc, 2004), y las aportadas por una maestra que actúa como informante clave al tener un mayor conocimiento sobre el contexto estudiado y un papel más influyente dentro del grupo-clase (Rodríguez Gómez et al., 1996), de veintiséis niños-as de 4-5 años de un colegio ubicado en el centro de Salamanca capital. Algunos de los ejemplos ofrecidos fueron ciertas palabras muy sugerentes como: genial, sorpresa y magia, o las mascotas preferidas: perro y gato (ver Tabla 1).

\begin{tabular}{|c|c|c|c|c|c|}
\hline \multicolumn{6}{|c|}{ PALABRAS RECOPILADAS DE DIVERSAS FUENTES } \\
\hline $\begin{array}{l}\text { FRECUEN } \\
\text { POBLACIÓ }\end{array}$ & $\begin{array}{l}\text { IES EN LA } \\
\text { INFANTIL }\end{array}$ & $\begin{array}{c}\text { POR } \\
\text { SUGERENCIA } \\
\text { DE MAESTRA }\end{array}$ & DE COLECCIÓ & "EL ZOO DE & CAS LETRAS" \\
\hline $\begin{array}{c}\text { CHOCOLATE } \\
\text { CIRCO } \\
\text { CORAZÓN } \\
\text { CROMO } \\
\text { CUENTO } \\
\text { DIBUJO } \\
\text { ESTRELLA } \\
\text { HELADO } \\
\text { JUGUETE } \\
\text { LUNA } \\
\text { MAMÁ } \\
\text { MARIPOSA }\end{array}$ & $\begin{array}{c}\text { ORDENADOR } \\
\text { PAPÁ } \\
\text { PARQUE } \\
\text { PELOTA } \\
\text { PEONZA } \\
\text { PISCINA } \\
\text { PLASTILINA } \\
\text { PULSERA } \\
\text { REGALO } \\
\text { TABLETA } \\
\text { TELEVISOR } \\
\text { ZUMO }\end{array}$ & $\begin{array}{c}\text { GATO } \\
\text { GENIAL } \\
\text { MAGIA } \\
\text { PEDO } \\
\text { PERRO } \\
\text { PIS } \\
\text { SORPRESA } \\
\text { YUPI }\end{array}$ & $\begin{array}{c}\text { ANILLO } \\
\text { ALEGRE } \\
\text { BICICLETA } \\
\text { BOCA } \\
\text { BOLSO } \\
\text { BONITA } \\
\text { CABAÑA } \\
\text { CABALLO } \\
\text { CANCIÓN } \\
\text { CEBRA } \\
\text { CIELO } \\
\text { CHARCOS } \\
\text { CHOCOLATE } \\
\text { CHUBASQUERO } \\
\text { DANZA } \\
\text { ESPADA } \\
\text { ESTRELLA } \\
\text { FELIZ } \\
\text { FIDEOS }\end{array}$ & $\begin{array}{c}\text { FLAN } \\
\text { FLORES } \\
\text { GALLETA } \\
\text { GIGANTE } \\
\text { GOLOSINAS } \\
\text { GLOBO } \\
\text { GUSANTTOS } \\
\text { JUGAR } \\
\text { KIOSKO } \\
\text { KIWI } \\
\text { KOALA } \\
\text { LECHE } \\
\text { LIBROS } \\
\text { LOBO } \\
\text { LUNA } \\
\text { LLUVIA } \\
\text { MARIPOSA } \\
\text { MONTAÑA } \\
\text { NIÑO-A }\end{array}$ & $\begin{array}{l}\text { NOCHE } \\
\text { NUBES } \\
\text { PALABRAS } \\
\text { PASTEL } \\
\text { PAPA } \\
\text { PIRATA } \\
\text { PINTURA } \\
\text { PLAYA } \\
\text { POMPAS } \\
\text { REGALIZ } \\
\text { ROSA } \\
\text { SALTAR } \\
\text { SOMBRERO } \\
\text { TORTILLA } \\
\text { ZANAHORIAS } \\
\text { ZAPATO } \\
\text { ZORRO } \\
\text { ZUMO }\end{array}$ \\
\hline
\end{tabular}

Tabla 1: Palabras recopiladas de diversas fuentes. Elaborado por García Rodríguez y García Díaz (2017)

Muchas de estas palabras originalmente recogidas serán eliminadas en la relación definitiva por su dificultad para ser representadas con imágenes fácilmente identificables.

\subsubsection{Análisis de los datos obtenidos}

El análisis de los datos cualitativos requiere cierto grado de sistematización (Rodríguez Gómez et al., 1996, 75) para lograr sintetizar y organizar los datos recopilados. En esta investigación se distinguen tres fases: (1) la reducción de datos según criterios temáticos, que lleva a (2) la categorización por familias temáticas de las palabras, puesto que «La categorización es una tarea simultánea a la separación en unidades cuando ésta se realiza atendiendo a criterios temáticos» (Rodríguez Gómez et al., 1996,208); y (3) la disposición en una tabla de doble entrada que muestra el orden de presentación de las consonantes clasificando las palabras en función del número y la complejidad silábica (Figura 2). 


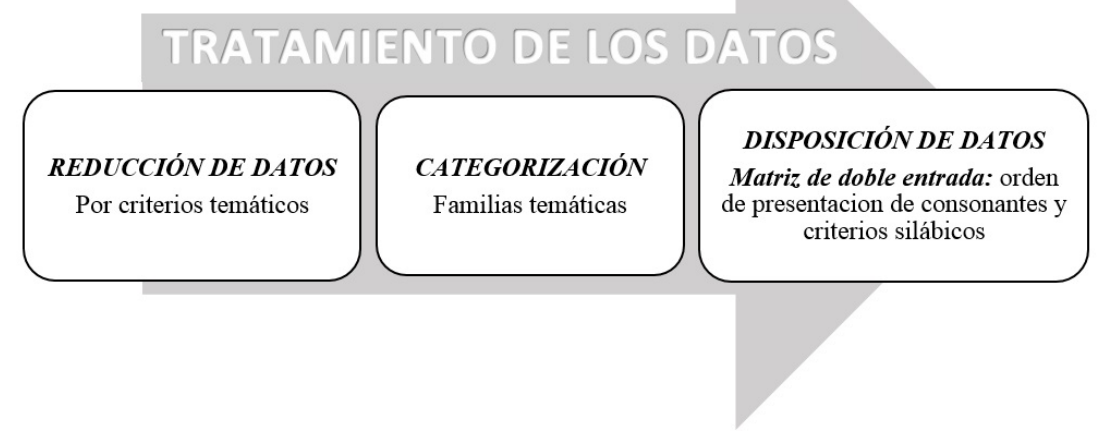

Figura 2: Fases del tratamiento de los datos. Elaborado por García Rodríguez y García Díaz (2017)

Los criterios utilizados para sintetizar y agrupar las palabras clara y organizadamente son temáticos y lingüísticos (atendiendo al número y la complejidad silábica). Las categorías temáticas se han definido a medida que se examinaban los datos, siguiendo un procedimiento inductivo, que origina familias temáticas. Se presentan en la Tabla 2 las dieciséis categorías temáticas resultantes, con la clasificación de todas las palabras recopiladas.

\begin{tabular}{|c|c|c|c|c|c|c|c|c|c|c|}
\hline \multicolumn{2}{|c|}{ ANIMALES } & \multicolumn{2}{|c|}{ ENTORNO/GEOGRAFIA } & \multicolumn{2}{|c|}{ ALIMENTOS } & $\begin{array}{l}\text { CONTEXTO } \\
\text { FAMIILIAR }\end{array}$ & \multicolumn{2}{|c|}{ ESCUELA } & \multicolumn{2}{|c|}{$\begin{array}{l}\text { MEDIOS DE } \\
\text { TRANSPORTE }\end{array}$} \\
\hline $\begin{array}{c}\text { GATO } \\
\text { PERRO } \\
\text { PATO } \\
\text { ZORRO } \\
\text { LOBO } \\
\text { OSO } \\
\text { CANGURO } \\
\text { GUSANO } \\
\text { ELEFANTE } \\
\text { MARIPOSA }\end{array}$ & $\begin{array}{c}\text { ABEJA } \\
\text { OVEJA } \\
\text { CEBRA } \\
\text { KOALA } \\
\text { DROMEDARIO } \\
\text { TIGRE } \\
\text { COCODRIL } \\
\text { TORTUGA } \\
\text { CIGÜENA } \\
\text { CABALLO }\end{array}$ & $\begin{array}{l}\text { NIEVE } \\
\text { LLUVIA } \\
\text { NOCHE } \\
\text { DIA } \\
\text { MAR } \\
\text { MONTANA } \\
\text { PUEBLO } \\
\text { CIUDAD } \\
\text { FLOR } \\
\text { ROSA } \\
\text { PISCINA } \\
\end{array}$ & $\begin{array}{c}\text { NUBE } \\
\text { CIELO } \\
\text { PLAYA } \\
\text { LUNA } \\
\text { SOL } \\
\text { ESTRELLA } \\
\text { SELVA } \\
\text { ARENA } \\
\text { ESPANAA } \\
\text { SALAMANCA }\end{array}$ & $\begin{array}{c}\text { ESPAGUETIS } \\
\text { LECHE } \\
\text { QUESO } \\
\text { MANZANA } \\
\text { PAN } \\
\text { HAMBURGUESA } \\
\text { BOCADILLO } \\
\text { ZUMO } \\
\text { REGALIZ } \\
\text { GOLOSINA } \\
\text { GELATINA }\end{array}$ & \begin{tabular}{|c|} 
TARTA \\
PATATAS \\
HELADO \\
CHOCOLATE \\
YOGUR \\
CHURROS \\
KIWI \\
GUSANITOS \\
CEREZA \\
FRESA \\
FLAN \\
COMER
\end{tabular} & $\begin{array}{c}\text { MAMA } \\
\text { PAPA } \\
\text { HERMANO/A } \\
\text { ABUELO/A } \\
\text { FAMIIIA } \\
\text { CASA } \\
\text { BEBE }\end{array}$ & $\begin{array}{c}\text { COLEGIO } \\
\text { PATIO } \\
\text { MESA } \\
\text { PIZARRA } \\
\text { ESTUCHE } \\
\text { MOCHILA } \\
\text { NINO/A } \\
\text { AMIGO/A } \\
\text { ESCRIBIR }\end{array}$ & $\begin{array}{l}\text { LAPIZ } \\
\text { GOMA } \\
\text { PINTURA } \\
\text { LIBRO } \\
\text { CUADERNO } \\
\text { DIBUJO } \\
\text { CANCION } \\
\text { EXCURSION } \\
\text { PALABRAS } \\
\text { LEER }\end{array}$ & $\begin{array}{r}\text { COC } \\
\text { AVI } \\
\text { BICIC } \\
\text { CAM } \\
\text { EXCAV } \\
\text { MO } \\
\text { BAR } \\
\text { YA }\end{array}$ & $\begin{array}{l}\text { HE } \\
\text { DN } \\
\text { ETA } \\
\text { ON } \\
\text { DORA } \\
\text { CO } \\
\text { CO } \\
\text { E }\end{array}$ \\
\hline \multicolumn{2}{|c|}{ PARTES DEL CUERPO } & \multicolumn{2}{|c|}{ PRENDAS DE VESTIR } & \multicolumn{2}{|c|}{ PROFESIONES } & $\begin{array}{l}\text { ESTADOS } \\
\text { DE ANIMO }\end{array}$ & \multicolumn{2}{|c|}{ FORMULAS SOCIALES } & \multicolumn{2}{|c|}{$\begin{array}{l}\text { PERSONAJES } \\
\text { FANTASTICOS }\end{array}$} \\
\hline $\begin{array}{c}\text { OJO } \\
\text { BOCA } \\
\text { NARIZ } \\
\text { DIENTE }\end{array}$ & $\begin{array}{l}\text { LENGUA } \\
\text { CABEZA } \\
\text { DEDO } \\
\text { CORAZON }\end{array}$ & \multicolumn{2}{|c|}{$\begin{array}{l}\text { ABRIGO } \\
\text { GUANTES } \\
\text { GORRO } \\
\text { ZAPATOS }\end{array}$} & \multicolumn{2}{|c|}{$\begin{array}{l}\text { BOMBERO/A } \\
\text { POLICIA } \\
\text { MEDICO/A } \\
\text { MAESTRO/A }\end{array}$} & $\begin{array}{c}\text { FELIZ } \\
\text { ALEGRE }\end{array}$ & \multicolumn{2}{|c|}{$\begin{array}{c}\text { HOLA } \\
\text { ADIOS } \\
\text { GRACIAS }\end{array}$} & \multicolumn{2}{|c|}{$\begin{array}{l}\text { BRUJA } \\
\text { MAGO } \\
\text { GIGANTE } \\
\text { GENIO } \\
\text { ANGEL }\end{array}$} \\
\hline \multicolumn{2}{|c|}{ TECNOLOGIAS } & \multicolumn{5}{|c|}{ INTERESES/OCIO } & \multicolumn{2}{|c|}{ ACCIONES } & \multicolumn{2}{|c|}{ OTROS } \\
\hline \multicolumn{2}{|c|}{$\begin{array}{c}\text { MOVIL } \\
\text { FOTO } \\
\text { ORDENADOR } \\
\text { CAMARA } \\
\text { VIDEO } \\
\text { TELEVISOR } \\
\text { PELICULA } \\
\text { SERIE } \\
\text { TABLETA }\end{array}$} & \multicolumn{2}{|c|}{$\begin{array}{c}\text { CROMO } \\
\text { PELOTA } \\
\text { COLUMPIO } \\
\text { TOBOGAN } \\
\text { COLCHONETA } \\
\text { GLOBO } \\
\text { FUTBOL } \\
\text { TESORO } \\
\text { PRISMATICOS }\end{array}$} & \multicolumn{2}{|c|}{$\begin{array}{c}\text { PIRATA } \\
\text { COCINITA } \\
\text { CUENTO } \\
\text { ESCONDITE } \\
\text { JUGUETE } \\
\text { MUNECA } \\
\text { PAYASO } \\
\text { YOYO }\end{array}$} & $\begin{array}{l}\text { FANTASMA } \\
\text { PUZLE } \\
\text { PLASTILINA } \\
\text { JUGAR } \\
\text { PINTAR } \\
\text { PEONZA } \\
\text { PALA } \\
\text { RASTRILLO }\end{array}$ & \multicolumn{2}{|c|}{$\begin{array}{c}\text { BAILAR } \\
\text { CANTAR } \\
\text { COMPRAR } \\
\text { HABLAR }\end{array}$} & $\begin{array}{c}\text { LLAVE } \\
\text { RELOJ } \\
\text { ALMOHADA } \\
\text { FLOTADOR }\end{array}$ & $\begin{array}{c}\text { REGALO } \\
\text { KIOSKO } \\
\text { XILOFONO } \\
\text { GUITARRA }\end{array}$ \\
\hline
\end{tabular}

Tabla 2: Clasificación de palabras por categorías temáticas. Elaborado por García Rodríguez y García Díaz (2017)

Por último se disponen los datos en una tabla que permite observar la cantidad de vocablos recopilados en función de varios criterios aportados por las características de la lengua - la diversidad fonológica, el número y la complejidad de las sílabas -, en los que se basará la selección de palabras generatrices (ver Anexo al final).

Para secuenciar las palabras atendiendo al grado de complejidad de las sílabas (de menor a mayor dificultad) se seguirá el orden siguiente: directas, inversas, mix- 
tas, trabadas y sinfones (Santos y Mateos, 1985; Cuetos Vega, 2008) y, por último, diptongos y palabras donde "y" actúa como semivocal (Santos y Mateos, 1985).

\subsubsection{Resultados}

La investigación cualitativa culmina con la presentación de los resultados (Rodríguez Gómez et al., 1996, 76).Se recopilan palabras que contienen todas las grafías de la lengua española, con la finalidad de seleccionar un conjunto de palabras generatrices que permitan trabajar todos los fonemas y sílabas de manera explícita.

Por relacionarse fácilmente con una imagen, las palabras recopiladas son, mayoritariamente, sustantivos (154 nombres comunes y 2 nombres propios), además de 9 verbos, 2 adjetivos y 3 interjecciones. La construcción temprana de frases, aproximadamente a partir de la sexta palabra, se favorece presentando los grafemas o letras "l" y "s" y los "comodines" "el", "la", "es" al comienzo del proceso didáctico.

En la clasificación de las palabras recopiladas y organizadas en categorías temáticas (Tabla 2), se advierte que las dieciséis categorías resultantes se pueden enmarcar dentro de las tres áreas de conocimiento del currículo infantil español especificadas en el Real Decreto 1630/2006 por el que se establecen las enseñanzas mínimas del segundo ciclo de la Educación Infantil (MEC, 2006) desarrolladas, posteriormente, por cada Comunidad autónoma en el currículo, en el caso de Castilla y León en el Decreto 122/2007, de 27 de diciembre (Consejería de Educación, 2008)de acuerdo con las que se categorizan las temáticas presentadas en la Tabla 3.

\begin{tabular}{|c|c|c|c|}
\hline $\begin{array}{c}\text { CONOCIMIENTO DE SÍ MISMO Y } \\
\text { AUTONOMIA PERSONAL }\end{array}$ & \multicolumn{2}{|c|}{ CONOCIMIENTO DEL ENTORNO } & $\begin{array}{c}\text { LENGUAJES: } \\
\text { COMUNICACIÓN Y } \\
\text { REPRESENTACIÓN }\end{array}$ \\
\hline INTERESES OCIO & ANIMALES & MEDIOS DE TRANSPORTE & \\
PARTES DEL CUERPO & NATURALEZA/GEOGRAFİ & PRENDAS DE VESTIR & TECNOLOGÍAS \\
ESTADO DE ÁNIMO & ALIMENTOS & PROFESIONES & FÓRMULAS SOCIALES \\
ACCIONES & CONTEXTO FAMILIAR & PERSONAJES FANTÁSTICOS & \\
& ESCUELA & OTROS & \\
\hline
\end{tabular}

Tabla 3: Clasificación de categorías temáticas en áreas de conocimiento. Elaborado por García Rodríguez y García Díaz (2017)

Mediante la elección de palabras se trabajan contenidos de todas las áreas del currículo, es decir, a través de las sesiones de lengua escrita se lleva a cabo una enseñanza globalizada.

\subsection{Conclusiones de la exploración del vocabulario infantil}

Algunos centros de interés de la población infantil de Salamanca se pueden generalizar a toda la infancia porque no se limitan al contexto concreto de la ciudad ni al periodo de edad en el que se ha centrado la investigación (de 4 a 7 años), como son:

$\sqrt{ }$ La influencia de las TIC: la infancia del siglo XXI vive inmersa en una sociedad tecnológica y tempranamente sabe manejar un móvil, un ordenador o una tableta.

$\sqrt{ }$ Las profesiones más populares suelen ser médico/a, bombero/a y maestro/a. 
$\sqrt{ }$ Entre los recursos de juego se diferencian pasatiempos temporales, como las series televisivas y las películas, y otros materiales de juego estables a lo largo del tiempo como: pelotas, cocinitas, muñecas, colecciones de cromos (cuya temática varía en relación a los pasatiempos pasajeros), personajes fantásticos como brujas, genios y magos o superhéroes, etc.

$\sqrt{ }$ Finalmente, los niños y niñas escolarizados están influidos por las temáticas de las unidades didácticas o proyectos curriculares que trabajan en el colegio.

Los datos analizados conducen a la conclusión de que la actualización del método de las palabras generatrices es necesaria para adecuarse a los intereses de la población infantil salmantina actual, $y$, teniendo en cuenta la influencia que ejercen las TIC, incorporar algún ejemplo de aparato electrónico presente en la vida diaria de los educandos.

\section{Nueva propuesta didáctica del método de palabras generatrices}

Partiendo del estudio realizado sobre el vocabulario de la población infantil de la ciudad de Salamanca, se realiza una selección de palabras y se presenta una lista de palabras generatrices actualizada junto al material necesario para poner en práctica el método propuesto por Freire, detallando la metodología a seguir.

Previamente se trabaja con las grafías vocálicas (las cinco vocales) de manera exhaustiva, hasta que los discentes las discriminen y reconozcan, tanto visual como fonológicamente. Para presentar cada vocal, se expondrá una palabra que empiece por la misma; así por ejemplo, la "a" se trabajará con "abanico" (y al mostrar la grafía de la palabra, se incidirá o se fijará la atención, solamente, en esta letra); la "e" con "elefante", la "i" con "imán", la "o" con "ojo" y la "u" con "uvas". Siempre que se pueda, se llevarán los objetos representados al aula (abanico, imán y uvas). Cuando no sea posible se utilizará una representación de las mismas (como un elefante de peluche) para fomentar la experimentación, el diálogo y los juegos del lenguaje. A partir de estas palabras podemos proponer actividades como buscar objetos del aula que empiecen con la vocal.

Una vez dominadas las vocales - proceso de aprendizaje que requiere de mucha dedicación y paciencia- comienza el trabajo propiamente dicho con el método de las palabras generatrices, mediante el cual se enseñan todos los fonemas consonánticos acompañados por los vocálicos, para facilitar su pronunciación y comprensión. En esta fase no es necesario presentar el nuevo fonema al inicio de la palabra, pues es superfluo para la discriminación y trabajo con las familias fonéticas.

Esta propuesta didáctica se enmarca en la Corriente Psicolingüística, ya que permite trabajar las dos vías de acceso a la lengua escrita: la vía léxica o de reconocimiento visual global de la palabra -cuyo uso se va incrementando con el hábito lector al enriquecer el léxico interno- y la vía fonológica o de asociación de cada grafema/letra con su fonema/sonido para llegar al significado de la palabra, predominante al comienzo del aprendizaje de la lengua escrita, al poseer un vocabulario interno más reducido. Aunque la lengua española es bastante transparente, la relación grafema-fonema no es unívoca; sin embargo, es el mecanismo más frecuente para acceder al significado de la palabra al enfrentarse a palabras desconocidas. 
También incorpora elementos de la Corriente Constructivista puesto que el alumnado analiza las palabras y crea autónomamente nuevos vocablos combinando los símbolos de forma creativa y descubriendo términos que comparte con el resto de compañeros-as, a los que explica su significado, lo que permite al aprendiz participar activamente en la construcción de su propio aprendizaje.

\subsection{Criterios para la selección de palabras}

En la Tabla 4 se pueden ver los criterios generales que se han tenido en cuenta para seleccionar el conjunto de palabras generatrices.

\begin{tabular}{|c|l|}
\hline $\begin{array}{c}\text { CRITERIO DE } \\
\text { SELECCIÓN }\end{array}$ & \multicolumn{1}{c|}{ EXPLICACIÓN } \\
\hline Riqueza fonética & $\begin{array}{l}\text { Considerando el orden frecuente de adquisición del sistema fonológico español., se adopta el } \\
\text { orden de presentación de consonantes propuesto por Monfort y Juárez (2010) y el sistema de } \\
\text { prevención de errores de estos mismos autores. }\end{array}$ \\
\hline $\begin{array}{c}\text { Evitar palabras } \\
\text { femeninas y masculinas }\end{array}$ & $\begin{array}{l}\text { Como por ejemplo, considerando las conclusiones obtenidas en la investigación, no se } \\
\text { contemplan las profesiones más populares entre la infancia. }\end{array}$ \\
\hline Categorías gramaticales & $\begin{array}{l}\text { Predominio de sustantivos, dado que a partir de la sexta palabra se comienzan a formar frases } \\
\text { con los "comodines" EL, LA y ES. }\end{array}$ \\
\hline $\begin{array}{c}\text { Palabras con matiz } \\
\text { educativo }\end{array}$ & $\begin{array}{l}\text { Se omiten los términos escatológicos y se incluyen nombres de frutas por estar relacionadas } \\
\text { con una alimentación saludable. }\end{array}$ \\
\hline $\begin{array}{c}\text { Clara relación imagen- } \\
\text { palabras }\end{array}$ & $\begin{array}{l}\text { Palabra que designen realidades concretas, que sean presentadas con imágenes atractivas, sin } \\
\text { detalles de fondo y fáciles de representar por el alumnado. }\end{array}$ \\
\hline Diversidad silábica & Contemplar las estructuras silábicas más frecuentes de la Lengua Española. \\
\hline $\begin{array}{c}\text { Capacidad } \\
\text { "generadora" }\end{array}$ & $\begin{array}{l}\text { Palabras que propicien la formación de numerosas palabras a partir de la combinación de sus } \\
\text { familias fonéticas. }\end{array}$ \\
\hline $\begin{array}{c}\text { Pertenecientes al } \\
\text { entorno próximo }\end{array}$ & $\begin{array}{l}\text { Palabras altamente motivantes e interesantes para los educandos (para propiciar aprendizajes } \\
\text { significativas). Deben ser palabras de su entorno próximo, que conozcan y sepan explicar a } \\
\text { los-as compañeros-as, ya que el diálogo en torno a cada palabra generatriz es una fase } \\
\text { fundamental del método. }\end{array}$ \\
\hline Presencia de las Tics & $\begin{array}{l}\text { Sensibilización ante la influencia que ejercen las Tecnologías de la Información y la } \\
\text { Comunicación en la sociedad actual y que supone un cambio en la metodología didáctica. }\end{array}$ \\
\hline $\begin{array}{c}\text { Evitar interpretaciones } \\
\text { sexistas }\end{array}$ & $\begin{array}{l}\text { En el caso de los juguetes infantiles, solamente se selecciona la palabra pelota frente a } \\
\text { muñecas o cocinitas. }\end{array}$ \\
\hline
\end{tabular}

Tabla 4: Criterios generales para la selección de palabras. Elaborado por García Rodríguez y García Díaz (2017).

\subsection{Cincuenta palabras generatrices}

En la Tabla 5 se recoge la lista de palabras generatrices seleccionadas a partir del estudio del vocabulario de la población infantil actual de Salamanca capital, secuenciadas en el orden de presentación que se ha considerado más apropiado para su aplicación didáctica. 


\begin{tabular}{|c|c|}
\hline SIILABAS DIRECTAS & SÍLABAS INVERSAS \\
\hline \multirow{26}{*}{$\begin{array}{ll}\text { 1. } & \text { MAMÁ } \\
\text { 2. } & \text { OSO } \\
\text { 3. } & \text { MESA } \\
\text { 4. } & \text { PAPÁ } \\
\text { 5. } & \text { PELOTA } \\
\text { 6. } & \text { LUNA } \\
\text { 7. } & \text { CASA } \\
\text { 8. } & \text { OVEJA } \\
\text { 9. } \text { MARIPOSA } \\
\text { 10. GELATINA } \\
\text { 11. ROSA } \\
\text { 12. REGALO } \\
\text { 13. ZUMO } \\
\text { 14. HELADO } \\
\text { 15. CEREZA } \\
\text { 16. CHOCOLATE } \\
\text { 17. CABALLO } \\
\text { 18. QUESO } \\
\text { 19. XILÓFONO } \\
\text { 20. PAYASO } \\
\text { 21. PERRO } \\
\text { 22. GUTTARRA } \\
\text { 23. KOALA } \\
\text { 24. KIWI } \\
\text { 25. CIGÜEÑA }\end{array}$} & $\begin{array}{l}\text { 26. ALMOHADA } \\
\text { 27. ANGEL }\end{array}$ \\
\hline & SIIABAS MIXTAS \\
\hline & 28. MANZANA \\
\hline & 29. TORTUGA \\
\hline & 30. PISCINA \\
\hline & 31. TELEVISOR \\
\hline & $\begin{array}{l}\text { 32. ORDENADOR } \\
\text { 33. ESPAGUETIS }\end{array}$ \\
\hline & SÍLABAS TRABADAS O SINFONES \\
\hline & 34. FLOTADOR \\
\hline & 35. FRESA \\
\hline & 36. TABLETA \\
\hline & 37. LIBRO \\
\hline & 38. GLOBO \\
\hline & 39. TIGRE \\
\hline & 40. COCODRILO \\
\hline & 41. ESTRELLA \\
\hline & 42. BIBICLETA \\
\hline & $\begin{array}{l}\text { 43. ESCRIBIR } \\
\text { 44. PLAYA }\end{array}$ \\
\hline & $\begin{array}{l}\text { 44. PLAYA } \\
\text { 45. PRISMÁTICOS }\end{array}$ \\
\hline & SÍLABA TRABADA Y MIXTA \\
\hline & 1. FLAN \\
\hline & Síl ABA CON "Y" \\
\hline & 2. REY \\
\hline & DIPTONGOS \\
\hline & $\begin{array}{ll}\text { 3. } & \text { NIEVE } \\
\text { 4. } & \text { FAMILIA }\end{array}$ \\
\hline & 5. CUENTO \\
\hline
\end{tabular}

Tabla 5: Propuesta de palabras generatrices. Elaborado por García Rodríguez y García Díaz (2017)

La nueva propuesta de palabras generatrices está formada por cincuenta términos en total, número que se encuentra entre las dos propuestas de los referentes más directos: el método original de Freire para la alfabetización de adultos, que contenía diecisiete palabras, y la adaptación para niños de Santos y Mateos (1985), con sesenta y dos.

Las palabras se secuencian teniendo en cuenta el orden más frecuente de adquisición del sistema fonológico español por parte de los educandos, de manera que al tiempo que se incluyan todas las grafías de la lengua, se considere la dificultad de pronunciación de ciertos fonemas como la $/ \mathrm{r} / \mathrm{o}$ las sílabas complejas con $/ 1 / \mathrm{y} / \mathrm{p} /$ (sinfones o sílabas trabadas), adoptando la propuesta de presentación de consonantes de Monfort y Juárez Sánchez (2010, 60), para evitar presentar consecutivamente letras o grafemas con algún parecido visual o fonético (Monfort y Juárez Sánchez, 2010, 61).

Los criterios generales utilizados para la secuenciación de palabras son los siguientes:

1. Complejidad de las estructuras silábicas: comenzar presentando estructuras silábicas más sencillas e ir avanzando hasta las más complejas: sílabas directas, inversas, trabadas... (Santos y Mateos, 1985; Cuetos Vega, 2008).

$\sqrt{ }$ Silabas directas: presentar todos los fonemas de la lengua española. 
$\checkmark$ Silabas inversas: presentar algunos ejemplos de familias fonéticas inversas bastante frecuentes en el vocabulario habitual, que tengan al mismo tiempo capacidad generadora para crear nuevas palabras con sus familias fonéticas.

$\checkmark$ Silabas mixtas: siguiendo la misma lógica que con las sílabas inversas.

$\checkmark$ Silabas trabadas: presentar los doce sinfones más frecuentes en la lengua española: FL, FR, BL, BR, GL, GR, DR, TR, CL, CR, PL y PR.

$\checkmark$ Sílabas más complejas y menos frecuentes (CCVC): incluir algún ejemplo para que los educandos sean conscientes de su existencia.

$\sqrt{ }$ Palabra con " $y$ " como semivocal: por la razón anterior.

$\sqrt{ }$ Diptongos: siguiendo el mismo razonamiento, se presentará algún ejemplo de diptongo, puesto que la explicación de las reglas ortográficas es más compleja y constituye un contenido propio de cursos posteriores.

2. Extensión silábica: contemplar palabras con diferente número de sílabas (monosílabas, bisílabas, trisílabas y polisílabas), teniendo en cuenta que cuanto más extensa es la palabra más complejo resulta el trabajo con sus familias fonéticas. Por ello, a la hora de secuenciar las palabras se comenzará con palabras bisílabas y, posteriormente, se irán incluyendo trisílabas, polisílabas y algún ejemplo de monosílabas (pocas, dada su escasa capacidad generadora) para que puedan ser conscientes de su existencia.

\subsection{Material del método de palabras generatrices}

En este apartado se detallan las decisiones metodológicas que conducen a la puesta en práctica del método en el aula, como la elección de la pauta caligráfica y el tipo de letra empleado para elaborar el material. A continuación, se justifican las razones que han motivado la elaboración de cada material y la metodología para su utilización en el aula.

La autonomía docente es esencial para adaptar el método a las circunstancias concretas de cada contexto de actuación educativa y a las características de cada grupo de educandos, pues la propuesta didáctica se ha diseñado con la finalidad de que pueda ajustarse a los intereses de la población infantil estimulando en ella la construcción activa de aprendizajes significativos y funcionales.

\subsubsection{Pauta caligráfica y tipo de letra utilizado en los materiales}

Con respecto a la pauta caligráfica, se considera conveniente dar alguna referencia espacial, dado que al escribir las palabras no sólo se aprende su grafía, sino también las características convencionales del texto escrito, como son la direccionalidad del trazo de izquierda a derecha y la horizontalidad de la escritura; por lo que se utiliza papel pautado de dos líneas ya que, al empezar a escribir, lo importante es que los aprendices sujeten bien el útil de escritura y se fijen en la grafía de las palabras, es decir, que comiencen a adquirir la melodía cinética de las palabras utilizando la letra cursiva (Santos y Mateos, 1985,41).

En cuanto al tipo de letra, el material está elaborado con letra cursiva o ligada, porque facilita la identificación de la palabra como unidad gramatical con sentido completo, es acorde con el pensamiento sincrético característico de la edad madu- 
rativa a la que se dirige y favorece la adquisición de una imagen visual global de la palabra (enriquecimiento del vocabulario interno).

\subsubsection{Material del método de palabras generatrices y metodología de trabajo}

Se confecciona el siguiente material:

1. Carteles de cada palabra generatriz (representación gráfica + palabra).

2. Fichas de trabajo individual de cada palabra generatriz, en las que se presentan las familias fonéticas que componen la palabra estudiada.

3. Una colección de familias fonéticas de madera (de todas las palabras seleccionadas), para facilitar la creación de nuevos vocablos mediante la combinación de las mismas.

En la figura 3 se presentan dos ejemplos de palabras generatrices con todo el material didáctico para el trabajo en el aula.

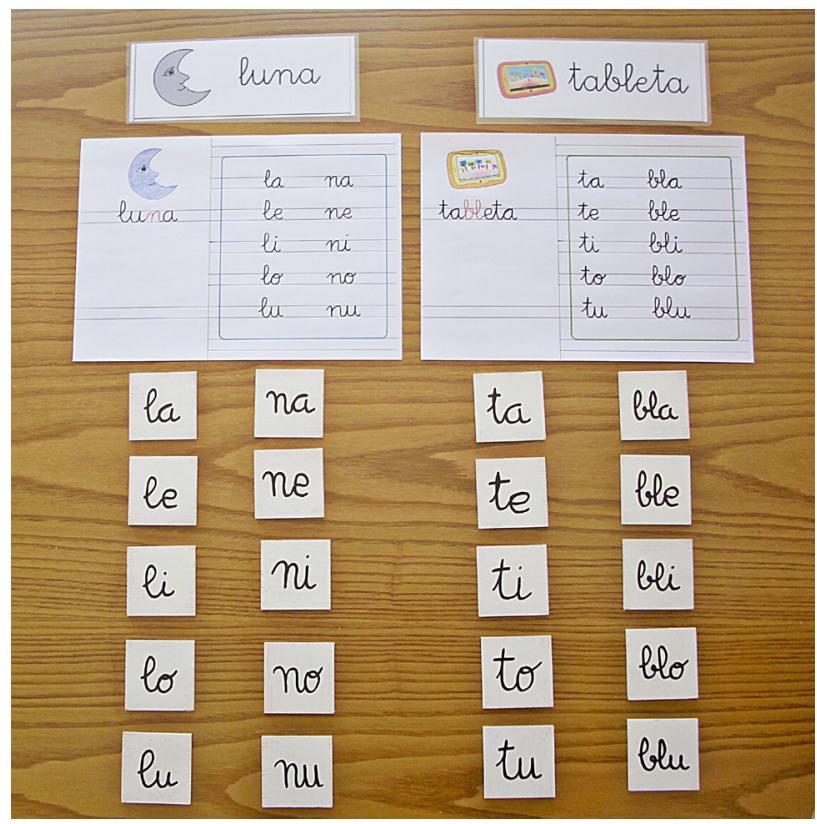

Figura 3: Dos palabras generatrices con material didáctico para poner en práctica el método. Elaborado por García Rodríguez y García Díaz (2017)

Se sugiere a continuación una posible metodología de trabajo en el aula.

a) En primer lugar se elaboran carteles plastificados de cada palabra generatriz, que, una vez utilizados, permanecerán en el aula durante todo el proceso de enseñanza-aprendizaje. Se irá presentando cada cartel según la secuencia de trabajo fijada, de forma que cada nueva palabra conocida sirva de modelo (se familiaricen con 
su grafía y comiencen a formar una imagen visual global de la misma), propicie el diálogo sobre lo que representa y constituya una referencia para identificar las familias fonéticas. Utilizar y manejar las familias fonéticas de madera, dialogando sobre ellas, servirá para crear nuevos vocablos a partir de sus combinaciones.

Finalmente, dichos carteles se colgarán en el aula (a una altura adecuada para que los-as niños-as puedan visualizarlos y manipularlos) y servirán como referentes clave para su aprendizaje, pues una vez que estén colgados varios carteles, se podrá dialogar sobre las semejanzas y diferencias entre las palabras, realizando juegos del lenguaje encaminados al desarrollo de la conciencia fonológica. Además, al verlos diariamente se refuerza la imagen visual global de la palabra (vía léxica) y se pueden comenzar a trabajar unidades de expresión más complejas y funcionales como las frases, ya que a partir de la sexta palabra se presentarán los "comodines" "el", "la" y "es".

b) Para trabajar cada palabra generatriz, también se elaboran fichas individuales de cada palabra. De esta forma, desde el primer día los aprendices comienzan a escribir: primero copian correctamente la palabra y dibujan su representación gráfica; observan las familias fonéticas de la palabra y crean nuevos vocablos (manipulando las familias fonéticas de madera), que se escribirán correctamente después de dialogar sobre su significado, de manera que todo el vocabulario nuevo sea comprendido.

Es fundamental resaltar la importancia de dar un modelo correcto de escritura de las palabras nuevas que surjan en las sesiones de lengua escrita. Es decir, intentar el aprendizaje sin error, evitando así una pregnancia ortográfica visual y grafomotriz incorrecta (Santos y Mateos, 1985, 30).Monfort y Juárez Sánchez (2010) trabajaron la enseñanza-aprendizaje de la lengua escrita española con alumnado que presentaba necesidades educativas especiales y descubrieron los beneficios del modelaje correcto (con este alumnado la repetición de modelos erróneos es más frecuente y corre el peligro de fijarse), siendo conveniente prevenir esa posible pregnancia visual o grafomotriz incorrecta con quienes acceden por primera vez al aprendizaje de la lengua escrita española. El objetivo es ofrecer un modelo correcto de escritura de la palabra; por ello, se escribirá en la pizarra y los aprendices la copiarán en sus hojas, interiorizando la correcta direccionalidad del trazo y la ortografía de la misma.

c) El tercer material elaborado son las familias fonéticas en madera. Trabajar la conciencia fonológica - aspecto de gran relevancia en la Corriente Psicolingüística requiere desarrollar la capacidad de manipular explícitamente los fonemas o sonidos de la lengua (Clemente Linuesa, 2008, 125). Las sílabas manipulables, fáciles de pronunciar y combinar, facilitan el desarrollo de la conciencia fonológica y elevan el rendimiento en lectura y escritura según un estudio realizado por Defior y Tudela (1994) con niños españoles de primer curso de Educación Primaria (Jiménez González y Ortiz González, 1995, 82-83).

Se trata de un material puramente manipulativo creado con la finalidad de facilitar la combinación de las familias fonéticas para formar nuevos vocablos, sobre todo al principio del método; de forma que los aprendices comprendan la lógica combinatoria y puedan progresar, haciendo mentalmente dichas combinaciones, una vez comprendido el mecanismo.

Con la utilización de este material se trabaja principalmente la conciencia silábica, que debe complementarse, durante todo el proceso de aprendizaje de la lengua escrita, con actividades metalingüísticas dirigidas a conseguir el desarrollo de la conciencia fonológica. 


\section{Consideraciones finales}

Enseñar a leer y a escribir es una hermosa tarea que va a transformar decisivamente la vida de quien aprende y que se proyecta en la sociedad de la que forma parte, puesto que, para su buen funcionamiento, es imprescindible el adecuado dominio de ese medio de comunicación, específicamente humano.

Es necesario tener en cuenta que se trata, muy probablemente, del aprendizaje académico más difícil que se consigue en los primeros años. Requiere de esfuerzo y tenacidad por parte de los discentes y de monumental paciencia, entre otras muchas virtudes, por parte de los docentes.

Por si quedara finalmente alguna duda, recordemos que el protagonismo en el proceso educativo lo ostenta el alumnado; que por excelente que se considere el método siempre se encuentra a expensas del estudiante -que posee un determinado ritmo y un peculiar estilo de aprendizaje que se ha de respetar-, siendo tarea del profesorado adecuar el método a las características que presenten quienes integren el grupo-clase.

Como consecuencia, dada la diversidad de las personas, solamente queda advertir que esta propuesta ha de ser aplicada en la práctica con la flexibilidad que cada contexto requiera y que cada docente habrá de explorar previamente. Quede bien claro que la autonomía didáctica docente es primordial a la hora de llevar a cabo la enseñanza de la lengua escrita con la actualización del método de Paulo Freire que proponemos. La investigación sobre el vocabulario de la población infantil de Salamanca, así como la metodología de trabajo sugerida, puede servir de ayuda y referencia, aunque claramente ha de ser adaptada a las peculiaridades de cada contexto y grupo-clase, o bien puede tomarse como orientación para la detección del vocabulario de otras poblaciones escolares.

\section{Bibliografía}

Alegría, Jesús (2006): "Por un enfoque psicolingüístico del aprendizaje de la lectura y sus dificultades -20 años después", Infancia y Aprendizaje, 29(1). Disponible en la página web: http:// www.researchgate.net/publication/39214652_Por_un_enfoque_psicolingüístico_del_aprendizaje_de_la_lectura_y_sus_dificultades_-20_años_después-[Consulta: 17 julio 2017].

Consejería de Educación (2008): Decreto 122/2007, de 27 de diciembre, por el que se establece el currículo de la Educación Infantil en la Comunidad de Castilla y León. Boletín Oficial de Castilla y León, ${ }^{\circ} 1$, de 2 de enero de 2008.

Clemente Linuesa, María (2008): Enseñar a leer. Bases teóricas y propuestas prácticas, Madrid, Ediciones Pirámide.

Cuetos Vega, Fernando (2008): Psicología de la lectura, Madrid, WK Educación.

Domínguez, Alberto y Cuetos Vega, Fernando (1992): "Desarrollo de las habilidades de reconocimiento de palabras en niños con distintas competencias lectoras", Cognitiva,4 (2). Disponible en la página web: http://dialnet.unirioja.es/servlet/articulo?codigo=122597[Consulta: 17 julio 2017].

Doumerc, Beatriz (2004): El zoo de las letras, Madrid, Bruño.

Ferreiro, Emilia y Teberosky, Ana (1979): Los sistemas de escritura en el desarrollo del niño, México, Siglo XXI. 
Freire, Paulo (1984, 9a ed.): Pedagogía del oprimido, Madrid, Siglo XXI de España Editores. Freire, Paulo (2002, $11^{\mathrm{a}}$ ed.): La educación como práctica de la libertad, Madrid, Siglo XXI. Freire, Paulo (2006, $2^{\mathrm{a}}$ ed.): Pedagogía de la indignación, Madrid, Morata.

García Rodríguez, María Luisa y Sánchez Gómez, María Cruz (2012):"El valor de la escritura", Papeles Salmantinos de Educación, 16,103-140.

Jiménez González, Juan Eugenioy Ortiz González, María del Rosario (1995): Conciencia fonológica y aprendizaje de la lectura: teoría, evaluación e intervención, Madrid, Síntesis.

Ministerio de Educación, Cultura y Deporte (2006): Real Decreto 1630/2006, de 29 de diciembre de 2006, por el que se establecen las enseñanzas mínimas del segundo ciclo de Educación Infantil. Boletín Oficial del Estado, nº 4 (185).

Monfort, Marc y Juárez Sánchez, Adoración (2010): Leer para hablar. La adquisición del lenguaje escrito en niños con alteraciones del desarrollo y/o del lenguaje, Madrid, Entha.

Rodríguez Gómez, Gregorio, Gil Flores, Javier y García Jiménez, Eduardo (1996): Metodología de la Investigación Cualitativa, Málaga, Aljibe.

Rondal, Jean-Adolphe(1982): El desarrollo del lenguaje, Barcelona, Médica y Técnica S.A.

Santos Asensi, María del Carmen y Mateos, Encarna (1985): Método de lectoescritura de Paulo Freire: Adaptación para niños, Salamanca, ICE-Universidad de Salamanca. 
Anexo: Clasificación de palabras por orden de presentación de consonantes y criterios silábicos (García Rodríguez y García Díaz, 2017).

\begin{tabular}{|c|c|c|c|c|c|}
\hline \multirow{2}{*}{ 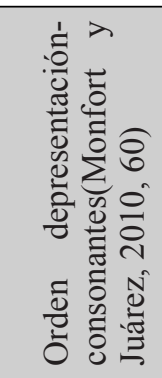 } & \multirow[b]{2}{*}{ 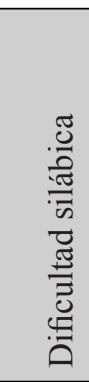 } & \multicolumn{4}{|c|}{ Número de sílabas } \\
\hline & & Monosílabas & Bisílabas & Trisílabas & Polisílabas \\
\hline \multirow{4}{*}{$\mathrm{m}$} & S.D. & & $\begin{array}{l}\text { MAMÁ, } \\
\text { MESA, } \\
\text { MAGO, } \\
\text { MOTO }\end{array}$ & $\begin{array}{l}\text { MÉDICO, MU- } \\
\text { ÑECA, MAÑA- } \\
\text { NA, MÚSICA }\end{array}$ & MARIPOSA \\
\hline & S.I. & & & MAESTRO & \\
\hline & S.M. & MAR & MÓVIL & $\begin{array}{l}\text { MOCHILA, } \\
\text { MANZANA, } \\
\text { MONTAÑA }\end{array}$ & \\
\hline & S.T. & & & & \\
\hline \multirow{4}{*}{$\mathrm{s}$} & S.D. & & SUMA, OSO & SUEÑO & \\
\hline & S.I. & & & $\begin{array}{l}\text { ESPAÑA, ESPA- } \\
\text { DA, ESTUCHE }\end{array}$ & $\begin{array}{l}\text { ESCONDI- } \\
\text { TE, ESPA- } \\
\text { GUETIS }\end{array}$ \\
\hline & S.M. & SOL & \begin{tabular}{|l} 
SELVA, \\
SALTAR \\
\end{tabular} & & $\begin{array}{l}\text { SALAMAN- } \\
\text { CA }\end{array}$ \\
\hline & S.T. & & & & \\
\hline \multirow{4}{*}{1} & S.D. & & $\begin{array}{l}\text { LOBO, } \\
\text { LUNA }\end{array}$ & & \\
\hline & S.I. & & & & $\begin{array}{l}\text { ALMOHA- } \\
\text { DA }\end{array}$ \\
\hline & S.M. & & $\begin{array}{l}\text { LÁPIZ, LE- } \\
\text { CHE }\end{array}$ & LENGUA & \\
\hline & S.T. & & & & \\
\hline \multirow{4}{*}{$\mathrm{p}$} & S.D. & & $\begin{array}{l}\text { PATO, PAPÁ, } \\
\text { PALA }\end{array}$ & $\begin{array}{l}\text { PELOTA, PAYA- } \\
\text { SO, PATIO, }\end{array}$ & POLICIA \\
\hline & S.I. & & & PEONZA & \\
\hline & S.M. & PAN & $\begin{array}{l}\text { PINTAR, } \\
\text { PUZLE }\end{array}$ & $\begin{array}{l}\text { PATATAS, PIS- } \\
\text { CINA, PINTU- } \\
\text { RA }\end{array}$ & $\begin{array}{l}\text { PLASTILI- } \\
\text { NA }\end{array}$ \\
\hline & S.T. & & $\begin{array}{l}\text { COMPRAR, } \\
\text { PLAYA }\end{array}$ & $\begin{array}{l}\text { PREMIO, PLÁ- } \\
\text { TANO, PRIS- } \\
\text { MÁTICOS }\end{array}$ & $\begin{array}{l}\text { PROFESO- } \\
\text { RA, CUM- } \\
\text { PLEAÑOS }\end{array}$ \\
\hline
\end{tabular}




\begin{tabular}{|c|c|c|c|c|c|}
\hline \multirow[t]{4}{*}{$\mathrm{rr}$} & S.D. & & $\begin{array}{l}\text { PERRO, } \\
\text { GORRO, } \\
\text { ZORRO, } \\
\text { CORRO, } \\
\text { ROSA }\end{array}$ & REGALO & \\
\hline & S.I. & & & & \\
\hline & S.M. & & RELOJ & REGALIZ & \\
\hline & S.T. & & & & \\
\hline \multirow{4}{*}{$\mathrm{t}$} & S.D. & & & \begin{tabular}{|l|} 
TESORO \\
\end{tabular} & \\
\hline & S.I. & & & & TELEVISOR \\
\hline & S.M. & & TARTA & $\begin{array}{l}\text { TOBOGÁN, } \\
\text { TORTUGA }\end{array}$ & \\
\hline & S.T. & & & $\begin{array}{l}\text { ESTRELLA,- } \\
\text { TRABAJO, } \\
\text { RASTRILLO, } \\
\text { FLOTADOR }\end{array}$ & \\
\hline \multirow{4}{*}{$\mathrm{n}$} & S.D. & & $\begin{array}{l}\text { NUBE, } \\
\text { NIÑO }\end{array}$ & $\begin{array}{l}\text { NIEVE (dipton- } \\
\text { go) }\end{array}$ & \\
\hline & S.I. & & & & \\
\hline & S.M. & & $\begin{array}{l}\text { NARIZ, } \\
\text { ÁNGEL }\end{array}$ & & \\
\hline & S.T. & & & & \\
\hline \multirow{4}{*}{$\begin{array}{l}\text { c } \\
\text { ca,co,cu }\end{array}$} & S.D. & & CASA & $\begin{array}{l}\text { CÁMARA, CA- } \\
\text { BEZA, }\end{array}$ & $\begin{array}{l}\text { COLEGIO, } \\
\text { PELICULA }\end{array}$ \\
\hline & S.I. & & & $\begin{array}{l}\text { CUENTO } \\
\text { (diptongo), CA- } \\
\text { MIÓN } \\
\end{array}$ & \\
\hline & S.M. & & $\begin{array}{l}\text { COMER, } \\
\text { CANTAR }\end{array}$ & $\begin{array}{l}\text { CANGURO, } \\
\text { CANCIÓN }\end{array}$ & $\begin{array}{l}\text { CUADER- } \\
\text { NO, CO- } \\
\text { LUMPIO, } \\
\text { COCINITA } \\
\end{array}$ \\
\hline & S.T. & & CROMO & ESCRIBIR & BICICLETA \\
\hline \multirow{4}{*}{$\mathrm{f}$} & S.D. & & FOTO & & $\begin{array}{l}\text { FAMILIA } \\
\text { (dipt.) }\end{array}$ \\
\hline & S.I. & & & & \\
\hline & S.M. & & $\begin{array}{l}\text { FELIZ, FÚT- } \\
\text { BOL }\end{array}$ & FANTASMA & \\
\hline & S.T. & FLOR, FLAN & FRESA & $\begin{array}{l}\text { FLAUTA (dip- } \\
\text { tongo) }\end{array}$ & \\
\hline
\end{tabular}




\begin{tabular}{|c|c|c|c|c|c|}
\hline \multirow{4}{*}{$r$} & S.D. & & & $\begin{array}{l}\text { ARENA, CÁ- } \\
\text { MARA, TESO- } \\
\text { RO, PIRATA } \\
\end{array}$ & MARIPOSA \\
\hline & S.I. & & & & $\begin{array}{l}\text { ORDENA- } \\
\text { DOR }\end{array}$ \\
\hline & S.M. & & & CARAMBA & \\
\hline & S.T. & & & & \\
\hline \multirow{4}{*}{$\mathrm{b} / \mathrm{v}$} & S.D. & & $\begin{array}{l}\text { BOCA, } \\
\text { BEBÉ, } \\
\text { BESO }\end{array}$ & $\begin{array}{l}\text { VÍDEO, ABE- } \\
\text { JA, CABALLO, } \\
\text { OVEJA }\end{array}$ & $\begin{array}{l}\text { BOCADI- } \\
\text { LLO }\end{array}$ \\
\hline & S.I. & & & AVIÓN & \\
\hline & S.M. & & $\begin{array}{l}\text { BARCO, } \\
\text { BALÓN }\end{array}$ & $\begin{array}{l}\text { BOMBERO, } \\
\text { BAILAR }\end{array}$ & \\
\hline & S.T. & & $\begin{array}{l}\text { BRUJA, } \\
\text { CEBRA, } \\
\text { HABLAR, } \\
\text { LIBRO }\end{array}$ & $\begin{array}{l}\text { ABRIGO, } \\
\text { ABRAZO, } \\
\text { PALABRA, } \\
\text { TABLETA }\end{array}$ & \\
\hline \multirow{4}{*}{$\mathbf{j}$} & S.D. & & OJO & & \\
\hline & S.I. & & & & \\
\hline & S.M. & & JUGAR & JUEGO & \\
\hline & S.T. & & & & \\
\hline \multirow{4}{*}{$z$} & S.D. & & $\begin{array}{l}\text { ZORRO, } \\
\text { ZUMO }\end{array}$ & PIZARRA & \\
\hline & S.I. & & & & \\
\hline & S.M. & & & ZAPATOS & \\
\hline & S.T. & & & & \\
\hline \multirow{4}{*}{$\mathrm{h}$} & S.D. & & HOLA & HELADO & \\
\hline & S.I. & & & & \\
\hline & S.M. & & & $\begin{array}{l}\text { HERMANO, } \\
\text { HORMIGA }\end{array}$ & $\begin{array}{l}\text { HAMBUR- } \\
\text { GUESA }\end{array}$ \\
\hline & S.T. & & & & \\
\hline \multirow{4}{*}{ d } & S.D. & & $\begin{array}{l}\text { DEDO, } \\
\text { DADO }\end{array}$ & DIBUJO & \\
\hline & S.I. & & & & \\
\hline & S.M. & DAR & & DIBUJAR & \\
\hline & S.T. & & DRAGÓN & & $\begin{array}{l}\text { COCODRI- } \\
\text { LO }\end{array}$ \\
\hline
\end{tabular}




\begin{tabular}{|c|c|c|c|c|}
\hline \multirow{4}{*}{$11 / y$} & S.D. & $\begin{array}{l}\text { YATE, } \\
\text { YOYÓ, LLA- } \\
\text { VE, REY }\end{array}$ & $\begin{array}{l}\text { LLUVIA, PA- } \\
\text { YASO }\end{array}$ & \\
\hline & S.I. & & & \\
\hline & S.M. & YOGUR & & \\
\hline & S.T. & & & \\
\hline \multirow{4}{*}{$\begin{array}{l}\text { g } \\
\text { ga,go,gu } \\
\text { gue, gui }\end{array}$} & S.D. & $\begin{array}{l}\text { GATO, GO- } \\
\text { RRO }\end{array}$ & GUSANO & GOLOSINA \\
\hline & S.I. & & $\begin{array}{l}\text { GUANTE, GUI- } \\
\text { TARRA }\end{array}$ & \\
\hline & S.M. & & GALLETAS & \\
\hline & S.T. & $\begin{array}{l}\text { GLOBO, } \\
\text { TIGRE }\end{array}$ & $\begin{array}{l}\text { GRACIAS, } \\
\text { ALEGRE }\end{array}$ & \\
\hline \multirow{4}{*}{$\begin{array}{l}\text { güe } \\
\text { güi }\end{array}$} & S.D. & & CIGÜEÑA & \\
\hline & S.I. & & & \\
\hline & S.M. & & PINGÜINO & \\
\hline & S.T. & & & \\
\hline \multirow{4}{*}{ ch } & S.D. & $\begin{array}{l}\text { NOCHE, } \\
\text { COCHE }\end{array}$ & & $\begin{array}{l}\text { CHOCOLA- } \\
\text { TE }\end{array}$ \\
\hline & S.I. & & & \\
\hline & S.M. & CHURROS & & \\
\hline & S.T. & & & \\
\hline \multirow{4}{*}{$\tilde{\mathrm{n}}$} & S.D. & $\begin{array}{l}\text { NIÑO-A, } \\
\text { PIÑA }\end{array}$ & & \\
\hline & S.I. & & & \\
\hline & S.M. & & & \\
\hline & S.T. & & & \\
\hline \multirow{4}{*}{$\mathrm{qu}$} & S.D. & QUESO & & \\
\hline & S.I. & & & \\
\hline & S.M. & $\begin{array}{l}\text { BOSQUE, } \\
\text { PARQUE }\end{array}$ & & \\
\hline & S.T. & & & \\
\hline \multirow{4}{*}{$\mathrm{x}$} & S.D. & & & XILÓFONO \\
\hline & S.I. & & & $\begin{array}{l}\text { EXCUR- } \\
\text { SIÓN, EX- } \\
\text { CAVADORA }\end{array}$ \\
\hline & S.M. & & & \\
\hline & S.T. & & & \\
\hline
\end{tabular}




\begin{tabular}{|c|c|c|c|c|}
\hline \multirow{4}{*}{$\mathrm{k}$} & S.D. & & $\begin{array}{l}\text { KOALA, KÁ- } \\
\text { RATE }\end{array}$ & \\
\hline & S.I. & & KIOSKO & \\
\hline & S.M. & & & \\
\hline & S.T. & & & $\begin{array}{l}\text { KILOGRA- } \\
\text { MO }\end{array}$ \\
\hline \multirow{4}{*}{$\begin{array}{l}\text { ce } \\
\text { ci }\end{array}$} & S.D. & CIELO & CEREZA & \\
\hline & S.I. & & & \\
\hline & S.M. & CIUDAD & & \\
\hline & S.T. & & & \\
\hline \multirow{4}{*}{$\begin{array}{l}\text { ge } \\
\text { gi }\end{array}$} & S.D. & & GENIO, MAGIA & GELATINA \\
\hline & S.I. & & & \\
\hline & S.M. & & $\begin{array}{l}\text { GIRASOL, } \\
\text { GIGANTE }\end{array}$ & \\
\hline & S.T. & & & \\
\hline \multirow{4}{*}{$\mathrm{w}$} & S.D. & KIWI, WII & & \\
\hline & S.I. & & & \\
\hline & S.M. & & & \\
\hline & S.T. & & & \\
\hline
\end{tabular}

- SD (Sílabas Directas), SI (Sílabas indirectas), SM (sílabas mixtas), ST (Sílabas trabadas).

Las palabras que presentan más de una estructura silábica compleja (sílaba inversa, mixta, trabada), se incluyen en el nivel o estructura de mayor complejidad, así, por ejemplo, ángel se incluye en la " $n$ " porque es interesante para trabajar la familia fonética [an, en, in, on, un] pero se incluye en "sílaba mixta" al ser la estructura silábica más compleja que contiene. 